\title{
Modified Multilag Methods for Volterra Functional Equations
}

\author{
By P. H. M. Wolkenfelt
}

\begin{abstract}
Linear multistep methods for ordinary differential equations in conjunction with a family of computationally efficient quadrature rules are employed to define a class of so-called multilag methods for the solution of Volterra integral and integro-differential equations. In addition, modified multilag methods are proposed which have the property that the stability behavior is independent of the choice of the quadrature rules. High order convergence of the methods is established. In particular, a special class of high order convergent methods is presented for the efficient solution of first-kind Volterra equations. Numerical experiments are reported.
\end{abstract}

1. Introduction. Consider the second-kind Volterra integral equation

$$
f(x)=g(x)+\int_{0}^{x} K(x, y, f(y)) d y, \quad 0 \leqslant x \leqslant X,
$$

whose kernel $K$ and forcing function $g$ are assumed to be sufficiently smooth.

In order to discretize (1.1) at $x=x_{n}$, we need an approximation of the Volterra integral operator at $x=x_{n}$. A conventional approach is to consider a family of quadrature rules W with weights $w_{n j}$ which yields the direct quadrature methods

$$
f_{n}=g\left(x_{n}\right)+h \sum_{j=0}^{n} w_{n j} K\left(x_{n}, x_{j}, f_{j}\right) .
$$

Here, $h$ denotes the step size, $x_{j}=j h$ are equidistant gridpoints, and $f_{j}$ denotes a numerical approximation to $f\left(x_{j}\right)$. A wide variety of specific methods (1.2) is discussed, e.g., in [2].

The stability behavior of a numerical method for (1.1) is analyzed by applying that method with a fixed positive step size $h$ to the test equation (cf. [3])

$$
f(x)=1+\lambda \int_{0}^{x} f(y) d y, \quad \lambda \in \mathbf{C} .
$$

Thus applying (1.2) to (1.3) yields the equations

$$
f_{n}=1+h \lambda \sum_{j=0}^{n} w_{n j} f_{j} .
$$

It is well known that the weights $w_{n j}$ frequently display a certain structure which makes it possible to reduce the discrete Volterra equation (1.4) to a finite term

Received July 8, 1981; revised March 17, 1982.

1980 Mathematics Subject Classification. Primary 65R20; Secondary 65D30.

Key words and phrases. Numerical analysis, Volterra integral and integro-differential equations, multilag methods, convergence and stability. 
recurrence relation. Particular attention has been paid (cf. [16], [20]) to the class of $(\rho, \sigma)$-reducible quadrature methods which have the property that the equations (1.4) reduce to the relations

$$
\sum_{i=0}^{k} a_{i} f_{n-i}=h \lambda \sum_{i=0}^{k} b_{i} f_{n-i}
$$

In (1.5) $a_{i}$ and $b_{i}$ represent the coefficients of a linear multistep (LM) method for ordinary differential equations (see e.g. [14]) which we shall denote by $(\rho, \sigma)$. Here, $\rho$ and $\sigma$ are polynomials defined as

$$
\rho(\zeta):=\sum_{i=0}^{k} a_{i} \zeta^{k-i}, \quad \sigma(\zeta):=\sum_{i=0}^{k} b_{i} \zeta^{k-i} .
$$

The main advantage of constructing methods for (1.1) which reduce to (1.5) lies in the fact that the stability behavior, determined by the stability polynomial $\rho(\zeta)-$ $h \lambda \sigma(\zeta)$, can be prescribed by choosing a suitable LM method. For example, the backward differentiation methods generate highly stable quadrature rules (cf. [20]). A disadvantage of $(\rho, \sigma)$-reducible quadrature methods, however, concerns their implementation. For instance, in the case of the backward differentiation methods just mentioned, either the weights must be generated numerically (cf. [20]) in each integration step which results in a rather awkward implementation and extra overhead costs, or the methods must be implemented following the imbedding approach described in [18] (see also Section 2) at the cost of a rather large number of additional arithmetic operations.

In this paper, we propose two new classes of methods which are more efficient than the $(\rho, \sigma)$-reducible quadrature methods since they can be constructed and implemented in a simple and straightforward fashion. The methods, which we have called multilag methods and modified multilag methods, are composed of an LM method $(\rho, \sigma)$ and a family of efficient quadrature rules $\mathcal{W}$.

It turns out, however, that the stability behavior of the multilag methods is not identical to that of the $(\rho, \sigma)$-reducible quadrature methods. In fact, stability is determined by $(\rho, \sigma)$ as well as by the quadrature rules* ${ }^{*}$. Adopting the idea of "modification" proposed by van der Houwen [11], [12] in connection with mixed Runge-Kutta methods for (1.1), we change the multilag methods by adding suitable perturbation terms (residuals) to obtain the modified multilag methods the stability behavior of which is determined only by $(\rho, \sigma)$ irrespective of the choice of the quadrature rules $\mathcal{W}$. As a result the modified multilag methods combine the advantages of the multilag methods and the $(\rho, \sigma)$-reducible quadrature methods. To be specific, the methods are easy to construct, simple to implement and computationally efficient. Monreover, they reduce to (1.5) when applied to (1.3).

The derivation of the multilag methods for (1.1) is essentially based on an appropriate approximation of the Volterra integral operator (see Section 2), and therefore it is not surprising that the same approximations can also be employed in connection with the numerical solution of other types of Volterra equations. To

\footnotetext{
${ }^{*}$ We intend to report on the stability behavior of the multilag methods for various choices of $\mathscr{W}$ in future work.
} 
demonstrate this, we shall apply our techniques also to derive numerical methods for Volterra integro-differential equations

$$
f^{\prime}(x)=F\left(x, f(x), \int_{0}^{x} K(x, y, f(y)) d y\right), \quad f(0)=f_{0},
$$

and for first-kind Volterra integral equations

$$
\int_{0}^{x} K(x, y, f(y)) d y=g(x), \quad g(0)=0 .
$$

We shall establish, in Sections 3 and 4, the order of convergence of the multilag methods as well as their modification for the solution of (1.1) and (1.7).

It is well known that for the solution of first-kind equations (1.8) by means of direct quadrature methods special stabilized quadrature rules must be constructed (see, e.g., [1], [6]). In Section 5, we shall present a class of high order convergent modified multilag methods which combine conventional quadrature rules with a highly stable LM method.

To illustrate the theoretical results we have included in Section 6 some numerical experiments with modified multilag methods in which we chose for $(\rho, \sigma)$ the highly stable backward differentiation methods and for $\mathcal{W}$ the Gregory quadrature rules.

2. Preliminaries and Notations. In this section we shall derive approximations of the Volterra integral operator $\int_{0}^{x} K(x, y, f(y)) d y$, which occurs in the functional equations (1.1), (1.7), and (1.8). For this derivation it is convenient to introduce the function $\Psi(t, x)$ defined as

$$
\Psi(t, x)=\int_{0}^{t} K(x, y, f(y)) d y,
$$

where (for the moment) $f$ is a given function. Following Pouzet (see, e.g., [2]), we regard $\Psi(t, x)$ as the solution of the differential equation (with parameter $x$ )

$$
\frac{\partial}{\partial t} \Psi(t, x)=K(x, t, f(t))
$$

with initial condition $\Psi(0, x)=0$. This observation suggests the use of methods for ordinary differential equations; cf. [9], [18]. Using an LM method $(\rho, \sigma)$ (with normalization $a_{0}=1$ ), we may define an approximation $\hat{\psi}_{n}(x)$ of $\psi_{n}(x)$ $\left(\psi_{n}(x):=\Psi(n h, x)\right)$ by the recurrence relation

$$
\hat{\psi}_{\nu}(x)=-\sum_{i=1}^{k} a_{i} \hat{\psi}_{\nu-i}(x)+h \sum_{i=0}^{k} b_{i} K\left(x, x_{\nu-i}, f\left(x_{\nu-i}\right)\right), \quad \nu=k(1) n
$$

provided that the starting values $\hat{\psi}_{0}(x), \ldots, \hat{\psi}_{k-1}(x)$ are given. In the treatment of second-kind Volterra equations Wolkenfelt et al. [18] discuss methods employing such approximations and indicate the equivalence with $(\rho, \sigma)$-reducible quadrature methods. A disadvantage of this approach is that, for the computation of $\hat{\psi}_{n}(x)$, the recurrence relation (2.3) must be evaluated for $\nu=k(1) n$, which may give a considerable amount of overhead, especially when dealing with systems of Volterra integral equations. This drawback can be avoided by the following approach: instead of defining starting values $\hat{\psi}_{0}(x), \ldots, \hat{\psi}_{k-1}(x)$ followed by a recursive evaluation of (2.3), we compute approximations $\tilde{\psi}_{n-k}(x), \ldots, \tilde{\psi}_{n-1}(x)$ by means of computationally efficient quadrature rules followed by one single application of $(\rho, \sigma)$ via $(2.3)$. 
To be specific, we define

$$
\hat{\psi}_{n}(x):=-\sum_{i=1}^{k} a_{i} \tilde{\psi}_{n-i}(x)+h \sum_{i=0}^{k} b_{i} K\left(x, x_{n-i}, f\left(x_{n-i}\right)\right),
$$

where

$$
\tilde{\psi}_{n}(x):=h \sum_{j=0}^{n} w_{n j} K\left(x, x_{j}, f\left(x_{j}\right)\right) .
$$

Here, $\mathscr{W}=\left\{w_{n j} \mid n \geqslant n_{0}, 0 \leqslant j \leqslant n\right\}$ denotes a family of quadrature rules. The value of $n_{0}$ depends on the accuracy of these rules. Obviously, (2.4) can only be applied for $n \geqslant n_{k}=n_{0}+k$.

Remark. Examples of computationally efficient quadrature rules are the rules with a finite repetition factor; see, e.g., [3]. In the case of a repetition factor of one, the weights satisfy

$$
w_{n j}-w_{n-1, j}= \begin{cases}0, & \text { if } 0 \leqslant j<n-\kappa, \\ \nabla w_{n j}, & \text { if } n-\kappa \leqslant j \leqslant n,\end{cases}
$$

so that $\tilde{\psi}_{n-k+1}(x), \ldots, \tilde{\psi}_{n-1}(x)$ defined in (2.5) can be computed recursively as follows

$$
\begin{aligned}
\tilde{\psi}_{m}(x)=\tilde{\psi}_{m-1}(x)+h \sum_{j=m-\kappa}^{m} \nabla w_{m j} K\left(x, x_{j}, f\left(x_{j}\right)\right) & \\
& m=n-k+1(1) n-1 .
\end{aligned}
$$

Specific examples are the Gregory quadrature rules [2]. It is easily verified that for the evaluation of $\hat{\psi}_{n}(x)$ by means of (2.4), (2.5), and (2.6) roughly $2 n k$ multiplications and additions are saved in comparison with (2.3).

So far we assumed that the function $f$ is known. Now assume that only approximations $f_{j}$ to $f\left(x_{j}\right)$ are available. In this case we replace in (2.4) and (2.5), $f\left(x_{j}\right)$ by $f_{j}$, $\hat{\psi}_{n}(x)$ by $\hat{I}_{n}(x)$ and $\tilde{\psi}_{n}(x)$ by $\tilde{I}_{n}(x)$ to obtain the approximations

$$
\hat{I}_{n}(x):=-\sum_{i=1}^{k} a_{i} \tilde{I}_{n-i}(x)+h \sum_{i=0}^{k} b_{i} K\left(x, x_{n-i}, f_{n-i}\right), \quad n \geqslant n_{k},
$$

where

$$
\tilde{I}_{n}(x):=h \sum_{j=0}^{n} w_{n j} K\left(x, x_{j}, f_{j}\right), \quad n \geqslant n_{0} .
$$

Since the function $\tilde{I}_{n}(x)$, which depends on all previously computed $f_{j}$-values, is usually called a lag term (or history term), we shall call the function $\hat{I}_{n}(x)$ a multilag approximation to $\psi_{n}(x)$.

For the convergence analysis of our methods we need the local truncation error $T_{n}(h ; x)$ of $(2.4)$ at $t=n h$ defined as

$$
\psi_{n}(x)=-\sum_{i=1}^{k} a_{i} \psi_{n-i}(x)+h \sum_{i=0}^{k} b_{i} K\left(x, x_{n-i}, f\left(x_{n-i}\right)\right)+T_{n}(h ; x) .
$$

Note that for an LM method of order $p$

$$
T_{n}(h ; x)=\left.e_{p+1} h^{p+1} \frac{\partial^{p}}{\partial t^{p}} K(x, t, f(t))\right|_{t=n h}+\Theta\left(h^{p+2}\right) \quad \text { as } h \rightarrow 0,
$$


where $e_{p+1} \neq 0$ denotes the error constant of $(\rho, \sigma)$; cf. [8]. For the rules (2.5) we define the quadrature error

$$
Q_{n}(h ; x):=\psi_{n}(x)-\tilde{\psi}_{n}(x) .
$$

Furthermore we assume that the quadrature weights $w_{n j}$ are uniformly bounded, i.e., $\left|w_{n j}\right| \leqslant \bar{w}$ for all $n$ and $j$.

In our theorems we shall establish a bound on the global discretization error in terms of quadrature errors, local truncation errors and errors in the starting values using the following notation:

$$
\begin{aligned}
& \delta_{1}(h)=\max \left\{\left|f\left(x_{j}\right)-f_{j}\right|: 0 \leqslant j \leqslant n_{0}-1\right\} \\
& \delta_{2}(h)=\max \left\{\left|f\left(x_{j}\right)-f_{j}\right|: n_{0} \leqslant j \leqslant n_{k}-1\right\} \\
& T_{N}(h)=\max \left\{\left|T_{n}\left(h ; x_{n}\right)\right|: n_{k} \leqslant n \leqslant N\right\} \\
& Q_{N}(h)=\max \left\{\left|Q_{n-i}\left(h ; x_{n}\right)\right|: n_{k} \leqslant n \leqslant N, 1 \leqslant i \leqslant k\right\}
\end{aligned}
$$

$$
\Delta Q_{N}(h)=\max \left\{\left|Q_{n-i}\left(h ; x_{n}\right)-Q_{n-i}\left(h ; x_{n-i}\right)\right|: n_{k} \leqslant n \leqslant N, 1 \leqslant i \leqslant k\right\}
$$

In order not to distract the reader's attention from the main results, all theorems are stated without proof. However, for those interested, the technical details can be found in the Appendix of [21].

3. Methods for Second-Kind Volterra Integral Equations. The second-kind Volterra equation (1.1) can be written as

$$
f(x)=g(x)+\Psi(x, x), \quad 0 \leqslant x \leqslant X,
$$

where we have used the notation (2.1).

3.1. Multilag Methods. In order to discretize (3.1) at $x=x_{n}$, we replace $f\left(x_{n}\right)$ by $f_{n}$ and $\Psi\left(x_{n}, x_{n}\right)=\psi_{n}\left(x_{n}\right)$ by $\hat{I}_{n}\left(x_{n}\right)$ defined in (2.7) to obtain the multilag method

$$
f_{n}=g\left(x_{n}\right)-\sum_{i=1}^{k} a_{i} \tilde{I}_{n-i}\left(x_{n}\right)+h \sum_{i=0}^{k} b_{i} K\left(x_{n}, x_{n-i}, f_{n-i}\right), \quad n \geqslant n_{k},
$$

where $\tilde{I}_{n}(x)$ is defined in (2.8). The required starting values are $f_{j}, j=0(1) n_{k}-1$.

For the global error $f\left(x_{n}\right)-f_{n}$ the following result can be derived.

THEOREM 3.1. Assume that $K$ satisfies the Lipschitz condition

$$
\left|K\left(x, y, \phi_{1}\right)-K\left(x, y, \phi_{2}\right)\right| \leqslant L_{1}\left|\phi_{1}-\phi_{2}\right| \text {. }
$$

Let $f(x)$ be the solution of (3.1) and let $f_{n}$ be defined by (3.2). Then for $h$ sufficiently $\operatorname{small}(X=N h)$

$$
\max _{n_{k} \leqslant n \leqslant N}\left|f\left(x_{n}\right)-f_{n}\right| \leqslant C \max \left\{h \delta_{1}(h), h \delta_{2}(h), Q_{N}(h), T_{N}(h)\right\},
$$

where $C$ is a constant independent of $N$ and $h$, and where $\delta_{1}(h), \delta_{2}(h), Q_{N}(h)$ and $T_{N}(h)$ are defined in (2.12) to (2.15). 
Using this theorem, high order convergence of the methods (3.2) is now readily established.

THEOREM 3.2. Let the condition (3.3) be satisfied, and assume that $g$ and $K$ are sufficiently smooth. In addition, let

(i) the LM method $(\rho, \sigma)$ be convergent of order $p$,

(ii) the quadrature rules W be of order $q$,

(iii) the errors in the starting values be of order s.

Then the multilag method (3.2) is convergent of order $r$, where

$$
r=\min \{s+1, q, p+1\} \text {. }
$$

To be specific

$$
\max _{n_{k} \leqslant n \leqslant N}\left|f\left(x_{n}\right)-f_{n}\right| \leqslant C h^{r} \quad \text { as } h \rightarrow 0, N \rightarrow \infty, N h=X,
$$

where $C$ is a constant independent of $N$ and $h$.

With respect to the stability analysis, we remark that the application of (3.2) to the basic test equation (1.3) yields the relations

$$
f_{n}=1-\sum_{i=1}^{k} a_{i} \tilde{I}_{n-i}+h \lambda \sum_{i=0}^{k} b_{i} f_{n-i}, \quad \tilde{I}_{n}=h \lambda \sum_{j=0}^{n} w_{n j} f_{j},
$$

which clearly indicates that the stability behavior of (3.2) depends on $(\rho, \sigma)$ as well as on the quadrature rules $\mathcal{W}$. Under suitable assumptions on the quadrature weights (e.g., reducibility [20] or finite repetition factor [3]) the relations (3.5) can be reduced to a recurrence relation in terms of $f_{n}$-values only and the stability behavior is then determined by a root condition on the associated stability polynomial. A systematic study along these lines for various choices of quadrature rules $थ$ will be the subject of future research.

In this paper we concentrate on a modification of (3.2) which has been constructed in such a way that the stability behavior with respect to (1.3) is independent of the choice of the quadrature rules $\mathcal{W}$ used for the lag terms $\tilde{I}_{n}(x)$.

3.2. Modified Multilag Methods. In [12] a modification of mixed Runge-Kutta methods was proposed (see also [11]) with the aim of improving the stability behavior. This modified method was derived by modifying the lag term by a suitable perturbation term which can be regarded as a residual; see [13]. Motivated by this approach, we present the following modification of (3.2)

$$
\begin{aligned}
f_{n}= & g\left(x_{n}\right)-\sum_{i=1}^{k} a_{i}\left\{\tilde{I}_{n-i}\left(x_{n}\right)+r_{n-i}\right\} \\
& +h \sum_{i=0}^{k} b_{i} K\left(x_{n}, x_{n-i}, f_{n-i}\right), \quad n \geqslant n_{k}, \\
r_{n} & =f_{n}-g\left(x_{n}\right)-\tilde{I}_{n}\left(x_{n}\right), \quad n \geqslant n_{0},
\end{aligned}
$$

where $\tilde{I}_{n}(x)$ is defined in (2.8). The modified multilag method (3.6) requires the starting values $f_{j}, j=0(1) n_{k}-1$. Note that $r_{n}$ defined in (3.6b) can be regarded as a residual.

We remark that the class (3.6) includes as a special case the methods proposed by van der Houwen [11] (who chose, in the notation (3.6), $a_{1}=-1, a_{2}=\cdots=a_{k}=0$ ). 
It is easily verified that application of (3.6) to the test equation (1.3) yields, due to cancellation of the $\tilde{I}_{n}$ terms, the recurrence relation (1.5). Thus the stability behavior of (3.6) is determined only by $(\rho, \sigma)$, and therefore identical to that of the $(\rho, \sigma)$-reducible quadrature methods.

Before establishing the high order convergence of the modified methods (3.6) we first state the following result.

THEOREM 3.3. Let $K$ satisfy the Lipschitz condition

$$
\begin{gathered}
\left|K\left(x, y, \phi_{1}\right)-K\left(x, y, \phi_{2}\right)-K\left(x_{n}, y, \phi_{1}\right)+K\left(x_{n}, y, \phi_{2}\right)\right| \\
\leqslant L_{1}^{*}\left|x-x_{n}\right|\left|\phi_{1}-\phi_{2}\right|,
\end{gathered}
$$

and let the LM method $(\rho, \sigma)$ be convergent. Furthermore let $f(x)$ be the solution of (3.1) and let $f_{n}$ be defined by (3.6). Then, for $h$ sufficiently small,

$$
\max _{n_{k} \leqslant n \leqslant N}\left|f\left(x_{n}\right)-f_{n}\right| \leqslant C \max \left\{h \delta_{1}(h), \delta_{2}(h), h^{-1} \Delta Q_{N}(h), h^{-1} T_{N}(h)\right\},
$$

where $C$ is a constant independent of $N$ and $h$ and where $\delta_{1}(h), \delta_{2}(h), \Delta Q_{N}(h)$ and $T_{N}(h)$ are defined in (2.12) to (2.16).

The Lipschitz condition (3.7) required in the above theorem is satisfied if, for example, $K_{x}$ satisfies a Lipschitz condition with respect to $f$. We then may write the left-hand side of (3.7) as

$$
\left|\int_{x_{n}}^{x}\left\{K_{x}\left(t, y, \phi_{1}\right)-K_{x}\left(t, y, \phi_{2}\right)\right\} d t\right|,
$$

from which the right-hand side of (3.7) is immediate. It can also be shown that $h^{-1} \Delta Q_{N}(h)$ has the same order of accuracy as $Q_{N}(h)$ provided that $K$ and $K_{x}$ are sufficiently smooth. This fact together with Theorem 3.3 yields

THEOREM 3.4. Let the assumptions of Theorem 3.3 and 3.2 be valid. Then the modified multilag method (3.6) is convergent of order $r^{*}$, where $r^{*}=\min \{s, q, p\}$.

Comparison of the results of Theorems 3.2 and 3.4 clearly shows the effect of the modification on the order of convergence: if $s \geqslant p+1$ and $q \geqslant p+1$, the modified methods may lose one order of accuracy. This result, although not rigorously proved here, has been verified numerically.

4. Methods for Volterra Integro-Differential Equations. Using (2.1), Eq. (1.7) can be written as

$$
f^{\prime}(x)=F(x, f(x), \Psi(x, x)), \quad 0 \leqslant x \leqslant X,
$$

with the initial condition $f(0)=f_{0}$. Application of an LM method for ordinary differential equations to (4.1) in which $\Psi\left(x_{n}, x_{n}\right)$ is replaced by a numerical approximation yields a wide class of numerical methods (cf. [5], [15], [16], [20]).

4.1 Multilag Methods. We shall employ a linear $k^{*}$-step method $\left(\rho^{*}, \sigma^{*}\right)$ with coefficients $a_{i}^{*}$ and $b_{i}^{*}$, and numerical approximations $\hat{I}_{n}=\hat{I}_{n}\left(x_{n}\right)$ as defined in (2.7) to obtain the methods

$$
\sum_{i=0}^{k} a_{i}^{*} f_{n-i}=h \sum_{i=0}^{k} b_{i}^{*} F\left(x_{n-i}, f_{n-i}, \hat{I}_{n-i}\right), \quad n \geqslant n_{k}=n_{0}+k
$$




$$
\begin{gathered}
\hat{I}_{n}=-\sum_{i=1}^{k} a_{i} \tilde{I}_{n-i}\left(x_{n}\right)+h \sum_{i=0}^{k} b_{i} K\left(x_{n}, x_{n-i}, f_{n-i}\right), \quad n \geqslant n_{k}, \\
\hat{I}_{n}=\tilde{I}_{n}\left(x_{n}\right) \text { if } n_{0} \leqslant n \leqslant n_{k}-1,
\end{gathered}
$$

where $\tilde{I}_{n}(x)$ is defined in (2.8). Note that we have assumed, without loss of generality, that $k^{*}=k$. The required starting values for $(4.2)$ are $f_{j}, j=0(1) n_{k}-1$.

A bound for the global discretization error is established in the following theorem.

THEOREM 4.1. Let $K$ satisfy the condition (3.3), and let $F$ satisfy the Lipschitz conditions

$$
\begin{aligned}
& \left|F\left(x, \phi_{1}, z\right)-F\left(x, \phi_{2}, z\right)\right| \leqslant L_{2}\left|\phi_{1}-\phi_{2}\right|, \\
& \left|F\left(x, \phi, z_{1}\right)-F\left(x, \phi, z_{2}\right)\right| \leqslant L_{3}\left|z_{1}-z_{2}\right|,
\end{aligned}
$$

and assume that the LM method $\left(\rho^{*}, \sigma^{*}\right)$ is convergent. Let $f(x)$ be the solution of (4.1) and let $f_{n}$ be defined by (4.2). Then for $h$ sufficiently small

$$
\begin{aligned}
& \max _{n_{k} \leqslant n \leqslant N}\left|f\left(x_{n}\right)-f_{n}\right| \\
& \leqslant C \max \left\{h \delta_{1}(h), \delta_{2}(h), h \delta_{3}(h), Q_{N}(h), T_{N}(h), h^{-1} T_{N}^{*}(h)\right\},
\end{aligned}
$$

where $C$ is a constant independent of $N$ and $h$ and where $\delta_{1}(h), \delta_{2}(h), Q_{N}(h)$ and $T_{N}(h)$ are defined in (2.12) to (2.15). Furthermore

$$
\begin{gathered}
\delta_{3}(h)=\max \left\{\left|Q_{n}\left(h ; x_{n}\right)\right|: n_{0} \leqslant n \leqslant n_{k}-1\right\}, \\
T_{N}^{*}(h)=\max \left\{\left|T_{n}^{*}\left(h ; x_{n}\right)\right|: n_{k} \leqslant n \leqslant N\right\},
\end{gathered}
$$

where $T_{n}^{*}\left(h ; x_{n}\right)$ denotes the local truncation error at $x=x_{n}$ of the LM method $\left(\rho^{*}, \sigma^{*}\right)$ when applied to (4.1).

An immediate consequence of the above theorem is

THEOREM 4.2. Let the conditions (3.3) and (4.3) be satisfied, and assume that $F$ and $K$ are sufficiently smooth. In addition, let

(i) the LM method $\left(\rho^{*}, \sigma^{*}\right)$ be convergent of order $p^{*}$,

(ii) the LM method $(\rho, \sigma)$ be convergent of order $p$,

(iii) the quadrature rules $\mathcal{W}$ be of order $q$,

(iv) the errors in the starting values be of order $s$.

Then the multilag method (4.2) is convergent of order $r$, where $r=\min \left\{s, q, p+1, p^{*}\right\}$.

Concerning the stability behavior, we note that the application of (4.2) to the basic test equation (cf. [16])

$$
f^{\prime}(x)=\xi f(x)+\eta \int_{0}^{x} f(y) d y, \quad \xi, \eta \in \mathbf{C},
$$

yields relations which depend also on the quadrature rules $\mathcal{W}$. In order to eliminate the effect of these quadrature rules on the stability behavior we construct a modification of (4.2).

4.2. Modified Multilag Methods. Along the same lines as in Subsection 3.2 we define the modified multilag methods by

$$
\sum_{i=0}^{k} a_{i}^{*} f_{n-i}=h \sum_{i=0}^{k} b_{i}^{*} F\left(x_{n-i}, f_{n-i}, \hat{I}_{n-i}\right), \quad n \geqslant n_{k},
$$




$$
\begin{gathered}
\hat{I}_{n}=-\sum_{i=1}^{k} a_{i}\left\{\tilde{I}_{n-i}\left(x_{n}\right)+r_{n-i}\right\}+h \sum_{i=0}^{k} b_{i} K\left(x_{n}, x_{n-i}, f_{n-i}\right), \quad n \geqslant n_{k} . \\
r_{n}=\hat{I}_{n}-\tilde{I}_{n}\left(x_{n}\right), \quad n \geqslant n_{k} .
\end{gathered}
$$

As in (4.2c) we define $\hat{I}_{n}=\tilde{I}_{n}\left(x_{n}\right)$ if $n_{0} \leqslant n \leqslant n_{k}-1$, which implies that $r_{n}=0$ if $n_{0} \leqslant n \leqslant n_{k}-1$.

Due to this modification, the method (4.8) applied to (4.7) yields the recurrence relations

$$
\begin{aligned}
& \sum_{i=0}^{k} a_{i}^{*} f_{n-i}=h \sum_{i=0}^{k} b_{i}^{*}\left(\xi f_{n-i}+\eta \hat{I}_{n-i}\right), \\
& \sum_{i=0}^{k} a_{i} \hat{I}_{n-i}=h \sum_{i=0}^{k} b_{i} f_{n-i} .
\end{aligned}
$$

Elimination of $\hat{I}_{n}$ yields a recurrence relation in $f_{n}$-values only whose characteristic (or stability) polynomial is given by

$$
\rho(\zeta)\left[\rho^{*}(\zeta)-h \xi \sigma^{*}(\zeta)\right]-h^{2} \eta \sigma(\zeta) \sigma^{*}(\zeta)
$$

which is independent of $\mathscr{W}$. Note that the same stability polynomials were found by Matthys [16] who considered $(\rho, \sigma)$-reducible quadrature rules.

We shall now deal with the convergence of (4.8). First we give the following bound for the global error.

THEOREM 4.3. Let $K$ satisfy the condition (3.7), and let $F$ satisfy (4.3), and let the LM methods $\left(\rho^{*}, \sigma^{*}\right)$ and $(\rho, \sigma)$ be convergent. Further, let $f(x)$ be the solution of $(4.1)$, and let $f_{n}$ be defined by (4.8). Then, for $h$ sufficiently small

$$
\begin{aligned}
& \max _{n_{k} \leqslant n \leqslant N}\left|f\left(x_{n}\right)-f_{n}\right| \\
& \quad \leqslant C \max \left\{h \delta_{1}(h), \delta_{2}(h), \delta_{3}(h), h^{-1} \Delta Q_{N}(h), h^{-1} T_{N}(h), h^{-1} T_{N}^{*}(h)\right\},
\end{aligned}
$$

where $C$ is a constant independent of $N$ and $h$, where $\delta_{1}(h), \delta_{2}(h), T_{N}(h)$ and $\Delta Q_{N}(h)$ are defined in (2.12) to (2.16), and where $\delta_{3}(h)$ and $T_{N}^{*}(h)$ are defined in (4.5), (4.6).

As a consequence we have

THEOREM 4.4. Let the assumptions of Theorem 4.3 and 4.2 be valid. Then the modified multilag method (4.8) is convergent of order $r^{*}$, where $r^{*}=\min \left\{s, q, p, p^{*}\right\}$.

From the results of Theorem 4.2 and 4.4 it is evident that the modified methods may lose one order of accuracy; cf. Subsection 3.2.

5. Modified Multilag Methods for First-Kind Volterra Integral Equations. In Sections 3 and 4 we considered general LM methods in conjunction with general quadrature rules. It turned out that convergent LM methods together with convergent quadrature rules generate convergent methods for second-kind Volterra equations and integro-differential equations.

It is well known, however, that for the solution of first-kind equations convergence of the quadrature rules does not generally imply convergence of the associated direct 
quadrature method and additional assumptions are necessary; see, e.g., [1], [6], [7], [10], [17], [19].

In this section we do not pursue complete generality and present the convergence results of a particular class of modified multilag methods. To be specific, we consider the methods

$$
\begin{gathered}
-\sum_{i=1}^{k} a_{i}\left\{\tilde{I}_{n-i}\left(x_{n}\right)+r_{n-i}\right\}+h b_{0} K\left(x_{n}, x_{n}, f_{n}\right)=g\left(x_{n}\right), \quad n \geqslant n_{k}, \\
r_{n}=g\left(x_{n}\right)-\tilde{I}_{n}\left(x_{n}\right), \quad n \geqslant n_{0},
\end{gathered}
$$

where $\tilde{I}_{n}(x)$ is defined in (2.8). The required starting values are $f_{j}, j=0(1) n_{k}-1$.

The methods (5.1) can be derived as follows. Using (2.1), the first-kind Volterra equation (1.8) can be written as

$$
\Psi(x, x)=g(x), \quad 0 \leqslant x \leqslant X .
$$

Discretization of (5.2) at $x=x_{n}$, using the approximation (2.7) in which we take $b_{2}=\cdots=b_{k}=0$, and modification by the "residual approach" then yields (5.1). Note that we have chosen a particular class of LM methods (i.e., $\sigma(\zeta)=b_{0} \zeta^{k}$ ) which includes the well-known backward differentiation methods. We emphasize that the quadrature rules $\mho$ are still free to choose.

For the global error the following bound can be derived.

THEOREM 5.1. In addition to the condition (3.7) assume that

$$
\left|K\left(x, x, \phi_{1}\right)-K\left(x, x, \phi_{2}\right)\right| \geqslant L_{4}\left|\phi_{1}-\phi_{2}\right| \quad\left(L_{4}>0\right) .
$$

Let the LM method $(\rho, \sigma)$ with $\sigma(\zeta)=b_{0} \zeta^{k}$ be convergent. Furthermore let $f(x)$ be the solution of (1.8), and let $f_{n}$ be defined by (5.1). Then for $h$ sufficiently small

$$
\max _{n_{k} \leqslant n \leqslant N}\left|f\left(x_{n}\right)-f_{n}\right| \leqslant C \max \left\{h \delta_{1}(h), h \delta_{2}(h), h^{-1} \Delta Q_{N}(h), h^{-1} T_{N}(h)\right\},
$$

where $C$ is a constant independent of $N$ and $h$, and where $\delta_{1}(h), \delta_{2}(h), \Delta Q_{N}(h)$ and $T_{N}(h)$ are defined in (2.12) to (2.16).

We remark that the Lipschitz condition (5.3) is implied by the sufficient conditions for the existence of a unique continuous solution to (1.8) given in [7]. To be specific, one of the conditions is that $|\partial K(x, x, f) / \partial f|$ should be bounded away from zero.

As an immediate consequence of Theorem 5.1 we have

THEOREM 5.2. Let the assumptions of Theorem 5.1 be valid and let $K$ and $g$ be sufficiently smooth. In addition, let

(i) the LM method $(\rho, \sigma)$ with $\sigma(\zeta)=b_{0} \zeta^{k}$ be convergent of order $p$,

(ii) the quadrature rules $\%$ be of order $q$,

(iii) the errors in the starting values be of order $s$.

Then the method (5.1) is convergent of order $r^{*}$, where $r^{*}=\min \{s+1, q, p\}$.

It is easily verified that the methods (5.1) applied to the test equation

$$
\int_{0}^{x} f(y) d y=g(x)
$$


reduce to $f_{n}=\left(h b_{0}\right)^{-1} \sum_{i=0}^{k} a_{i} g\left(x_{n-i}\right)$, irrespective of the choice of the quadrature rules थ. As a result, the methods (5.1) correspond to "local differentiation formulae" which is a desirable property with respect to stability (see e.g. [17, p. 417]).

6. Numerical Experiments. In this section we report on numerical experiments with modified multilag methods (3.6), (4.8) and (5.1). For the LM method $(\rho, \sigma)$ and the quadrature rules $\mathscr{W}$ we chose, for $p=2(1) 6$, the $p$ th order backward differentiation (BD) methods [14] and the $p$ th order Gregory quadrature rules, respectively. In the methods (4.8) we took $\left(\rho^{*}, \sigma^{*}\right)$ identical to $(\rho, \sigma)$. The methods are denoted by BDG $p(p=2(1) 6)$.

The methods were applied to test problems (taken from [5], [6] and [20]) with known exact solution. Integration was performed with a constant step size, and the necessary starting values were computed from the exact solution. In consequence of the Theorems 3.4, 4.4 and 5.2 the methods BDG $p$ are of order $p$, asymptotically.

In the tables of results we have tabulated for different orders and a sequence of stepsizes, the number of correct decimal digits cd (defined by $-{ }^{10} \log$ (absolute error)) at the endpoint of integration. Moreover we have listed in the convergence experiments the computed order $p^{*}\left(\right.$ defined by $\left.\{\operatorname{cd}(h)-\operatorname{cd}(2 h)\} /{ }^{10} \log 2\right)$.

All calculations have been performed on a CDC CYBER 750 installation using 14 significant digits.

6.1. Second-Kind Volterra Integral Equations. In order to test their high order convergence, we have applied the BDG methods to the following problem

$$
f(x)=\frac{1}{2} x^{2} \exp (-x)+\frac{1}{2} \int_{0}^{x}(x-y)^{2} \exp (y-x) f(y) d y, \quad 0 \leqslant x \leqslant 6,
$$

with exact solution $f(x)=\frac{1}{3}-\frac{1}{3} \exp (-3 x / 2)\left\{\cos \left(\frac{1}{2} x \sqrt{3}\right)+\sqrt{3} \sin \left(\frac{1}{2} x \sqrt{3}\right)\right\}$. In Table 6.1.1 the results are tabulated for various choices of $h$.

TABLE 6.1.1

Number of correct digits at $x=6$ and the computed order $p^{*}$ for the BDG methods applied to (6.1.1)

\begin{tabular}{|c|c|c|c|c|c|}
\hline$h^{-1}$ & $p=2$ & $p=3$ & $p=4$ & $p=5$ & $p=6$ \\
\hline \multirow[t]{2}{*}{4} & 1.89 & 1.86 & 2.34 & 2.97 & 3.51 \\
\hline & 1.1 & 2.4 & 3.0 & 4.1 & 4.7 \\
\hline \multirow[t]{2}{*}{8} & 2.22 & 2.57 & 3.25 & 4.21 & 4.92 \\
\hline & 1.6 & 2.7 & 3.5 & 4.6 & 5.4 \\
\hline \multirow[t]{2}{*}{16} & 2.70 & 3.37 & 4.31 & 5.60 & 6.55 \\
\hline & 1.8 & 2.9 & 3.8 & 4.8 & 5.8 \\
\hline \multirow[t]{2}{*}{32} & 3.25 & 4.23 & 5.44 & 7.05 & 8.28 \\
\hline & 1.9 & 2.9 & 3.9 & 4.9 & 6.1 \\
\hline 64 & 3.83 & 5.11 & 6.61 & 8.53 & 10.10 \\
\hline
\end{tabular}

From this table it is obvious that the computed order tends to the theoretical order of convergence. 
The favorable stability behavior of the BDG methods is demonstrated in the following example:

$$
f(x)=g(x)-\lambda \int_{0}^{x} \frac{1+x}{1+y} f^{2}(y) d y, \quad x \geqslant 0,
$$

with exact solution $f(x)=[1+(1+x) \exp (-x)]^{1 / 2}$ if we choose $g(x)=f(x)+$ $\lambda(1+x)[\ln (1+x)+1-\exp (-x)]$. We considered the values $\lambda=1,10,100,1000$ and 10000 which makes (6.1.2) increasingly stiff. The endpoint of integration was 192h. The results are given in Table 6.1.2.

TABLE 6.1.2

The number of correct digits at $x=192 h$ for problem (6.1.2)

\begin{tabular}{|rrrcccr|}
\hline$h^{-1}$ & $\lambda$ & $p=2$ & $p=3$ & $p=4$ & $p=5$ & $p=6$ \\
\hline & 1 & 3.23 & 3.83 & 4.97 & 5.10 & 5.84 \\
& 10 & 3.23 & 3.84 & 4.98 & 5.11 & 5.85 \\
4 & 100 & 3.24 & 3.84 & 4.98 & 5.11 & 5.85 \\
& 1000 & 3.24 & 3.84 & 4.98 & 5.11 & 5.85 \\
& 10000 & 3.24 & 3.84 & 4.98 & 5.11 & 5.85 \\
& 1 & 3.84 & 4.93 & 6.19 & 7.01 & 8.21 \\
& 10 & 3.87 & 4.96 & 6.22 & 7.04 & 8.24 \\
16 & 100 & 3.87 & 4.97 & 6.23 & 7.05 & 8.24 \\
& 1000 & 3.87 & 4.97 & 6.23 & 7.05 & 8.24 \\
& 10000 & 3.87 & 4.97 & 6.23 & 7.05 & 8.24 \\
& 1 & 5.18 & 6.41 & 8.06 & 9.29 & 10.46 \\
& 10 & 4.99 & 6.42 & 8.08 & 9.35 & 10.65 \\
64 & 100 & 4.99 & 6.42 & 8.09 & 9.37 & 10.36 \\
& 1000 & 4.99 & 6.42 & 8.09 & 9.43 & 10.63 \\
& 10000 & 4.99 & 6.42 & 8.09 & 9.40 & 10.44 \\
\hline
\end{tabular}

The results show that for fixed $h$ the accuracy is hardly affected by increasing stiffness and justify the conclusion that the BDG methods are highly-stable.

6.2. Volterra Integro-Differential Equations. To test the high order convergence we applied the BDG methods to

$$
\begin{aligned}
f^{\prime}(x)= & -x-(1+x)^{-2}+\frac{1}{f(x)} \ln \frac{2+2 x}{2+x} \\
& +\int_{0}^{x} \frac{d y}{1+(1+x) f(y)}, \quad 0 \leqslant x \leqslant 10 .
\end{aligned}
$$

Taking $f(0)=1$ yields the exact solution $f(x)=(1+x)^{-1}$. The results summarized in Table 6.2.1 clearly show that the computed order tends to the theoretical order of convergence, except for the sixth order method. 
TABLE 6.2.1

Number of correct digits at $x=10$ and computed order $p^{*}$ for the BDG methods applied to (6.2.1)

\begin{tabular}{|c|c|c|c|c|c|}
\hline$h^{-1}$ & $p=2$ & $p=3$ & $p=4$ & $p=5$ & $p=6$ \\
\hline \multirow[t]{2}{*}{4} & 5.85 & 5.76 & 6.32 & 7.00 & 7.60 \\
\hline & 0.8 & 1.4 & 1.8 & 2.0 & 2.3 \\
\hline \multirow[t]{2}{*}{8} & 6.10 & 6.19 & 6.86 & 7.59 & 8.30 \\
\hline & 1.0 & 2.2 & 2.7 & 3.2 & 3.4 \\
\hline \multirow[t]{2}{*}{16} & 6.40 & 6.84 & 7.67 & 8.51 & 9.33 \\
\hline & 1.6 & 2.6 & 3.3 & 3.9 & 4.4 \\
\hline \multirow[t]{2}{*}{32} & 6.89 & 7.61 & 8.65 & 9.67 & 10.65 \\
\hline & 1.9 & 2.8 & 3.6 & 4.3 & 7.1 \\
\hline 64 & 7.45 & 8.45 & 9.73 & 10.97 & 12.79 \\
\hline
\end{tabular}

For the stability test we applied the methods to

$$
\left\{\begin{array}{l}
f^{\prime}(x)=[d(x)-\alpha f(x)-\beta z(x)]^{3}-1, \quad f(0)=1 \\
z(x)=\int_{0}^{x}(x+\gamma y)^{\delta} f^{3}(y) d y
\end{array}\right.
$$

Choosing $d(x)=1+\alpha+\gamma^{-1}(1+\delta)^{-1} \beta x^{\delta+1}\left\{(1+\gamma)^{\delta+1}-1\right\}$ yields the exact solution $f(x) \equiv 1$. As in [20] we considered the values $\alpha=40, \beta=15, \gamma=2$, and $\delta=3 / 2$, and integration was performed with $h=1 / 8$. On the basis of the stability regions of the $\mathrm{BDG}$ methods (which are identical to those of the [BD; $\mathrm{BD}]$ methods given in [20]), we expect the methods to yield stable results. In Table 6.2.2 the results are given at some gridpoints.

TABLE 6.2 .2

Number of correct digits for problem (6.2.2) obtained with the BDG methods with $h=1 / 8$

\begin{tabular}{|rccccc|}
\hline$x$ & $p=2$ & $p=3$ & $p=4$ & $p=5$ & $p=6$ \\
\hline 1.0 & 3.23 & 4.37 & 5.44 & 6.17 & $*$ \\
3.0 & 4.25 & 6.07 & 6.72 & 8.54 & 7.72 \\
5.0 & 4.45 & 6.93 & 7.06 & 8.47 & 8.25 \\
7.0 & 4.60 & 7.49 & 7.28 & 8.68 & 8.15 \\
16.0 & 5.00 & 8.15 & 7.79 & 9.23 & 9.82 \\
\hline
\end{tabular}

The asterisk in this table indicates that $x=1$ is a point where an exact starting value was given. The numerical results clearly display the stable behavior of the BDG methods.

6.3. First-Kind Volterra Integral Equations. We applied the BDG methods to the following problems taken from [6]

$$
2 \int_{0}^{x} \cos (x-y) f(y) d y=\exp (x)+\sin (x)-\cos (x),
$$




$$
\int_{0}^{x} \exp (y-x) f(y) d y=\sinh (x) .
$$

Both problems have the exact solution $f(x)=\exp (x)$. The endpoint of integration was $x=4$. The correct order of convergence of the BDG methods up to order five is shown by the Tables 6.3.1 and 6.3.2.

TABLE 6.3.1

Number of correct digits at $x=4$ and the computed order $p^{*}$

of the $B D G$ methods applied to (6.3.1)

\begin{tabular}{|c|c|c|c|c|c|}
\hline$h^{-1}$ & $p=2$ & $p=3$ & $p=4$ & $p=5$ & $p=6$ \\
\hline 10 & 0.87 & 1.50 & 2.20 & 3.20 & 4.55 \\
\hline & 2.3 & 2.8 & 3.9 & 4.9 & 6.6 \\
\hline 20 & 1.55 & 2.33 & 3.36 & 4.68 & 6.54 \\
\hline 40 & 2.20 & 3.20 & 4.54 & 6.18 & 8.50 \\
\hline 80 & $2.83^{2.1}$ & $4.09^{3.0}$ & $5.73^{4.0}$ & $7.68^{5.0}$ & $9.54^{3.5}$ \\
\hline
\end{tabular}

TABLE 6.3.2

Number of correct digits at $x=4$ and computed order $p^{*}$ of the BDG methods applied to (6.3.2)

\begin{tabular}{|c|c|c|c|c|c|}
\hline$h^{-1}$ & $p=2$ & $p=3$ & $p=4$ & $p=5$ & $p=6$ \\
\hline 10 & -0.02 & 0.81 & 1.64 & 2.45 & 1.81 \\
\hline & 1.9 & 2.8 & 3.8 & 4.7 & 1.4 \\
\hline 20 & 0.54 & 1.66 & 2.77 & 3.87 & 2.23 \\
\hline 40 & 1.12 & 2.54 & 3.94 & 5.32 & 6.40 \\
\hline 80 & 1.71 & 3.43 & 5.12 & 6.80 & 8.43 \\
\hline
\end{tabular}

Although not displayed in the tables of results, the global error turns out to be a smooth function except for the sixth order method when $h$ is small $(h=1 / 40,1 / 80)$. This may explain the uncertain behavior of BDG6.

7. Concluding Remarks. The results of Section 6 justify the conclusion that the construction presented in this paper yields high order convergent methods which can be made highly stable by choosing a highly stable LM method.

To emphasize, we repeat that the modified multilag methods applied to the basic test equations of (1.1), (1.7), and (1.8) yield exactly the same stability polynomials as those obtained with $(\rho, \sigma)$-reducible quadrature methods. As a consequence, all 
stability results previously derived for $(\rho, \sigma)$-reducible quadrature methods (e.g., $A$-stability results [16], stability regions [4], [5],[20]) also hold for the modified multilag methods.

Finally we remark that the class of methods presented here can easily be extended by considering cyclic LM methods for ordinary differential equations. In this case the method (3.6) for example takes the form

$$
f_{n}=g\left(x_{n}\right)-\sum_{i=1}^{k} a_{i}^{(n)}\left\{\tilde{I}_{n-i}\left(x_{n}\right)+r_{n-i}\right\}+h \sum_{i=0}^{k} b_{i}^{(n)} K\left(x_{n}, x_{n-i}, f_{n-i}\right),
$$

with $r_{n}$ defined as in (3.6b) and where $a_{i}^{(n)}$ and $b_{i}^{(n)}$ are periodic functions of $n$. The proof of high order convergence of (7.1) will probably be more complicated than for the methods presented in this paper. On the other hand, the stability properties of cyclic LM methods are well known for ODE-theory and thus can be exploited to construct, in a straightforward fashion, highly accurate, highly stable modified multilag methods for the efficient solution of Volterra equations.

Acknowledgements. The author would like to thank Professor Dr. P. J. van der Houwen for his constructive remarks and valuable advice. He is also grateful to Mrs. Joke Blom, who carried out the computations summarized in Section 6 of this paper.

Department of Numerical Mathematics

Mathematisch Centrum

Kruislaan 413

1098 SJ Amsterdam, The Netherlands

1. C. ANDrade \& S. MCKeE, "On optimal high accuracy linear multistep methods for first kind Volterra integral equations," BIT, v.19, 1979, pp. 1-11.

2. C. T. H. BAKER, The Numerical Treatment of Integral Equations, Clarendon Press, Oxford, 1977.

3. C. T. H. BAKER \& M. S. KEECH, "Stability regions in the numerical treatment of Volterra integral equations," SIAM J. Numer. Anal., v. 15, 1978, pp. 394-417.

4. C. T. H. Baker, A. Makroglou \& E. Short, "Regions of stability in the numerical treatment of Volterra integro-differential equations," SIAM J. Numer. Anal., v. 16, 1979, pp. 890-910.

5. H. BRUNNER \& J. D. LAMBERT, "Stability of numerical methods for Volterra integro-differential equations," Computing, v. 12, 1974, pp. 75-89.

6. C. J. GlaDwin, "Quadrature rule methods for Volterra integral equations of the first kind," Math. Comp., v. 33, 1979, pp. 705-716.

7. C. J. GlaDwin \& R. JelTSCH, "Stability of quadrature rule methods for first kind Volterra integral equations," BIT, v. 14, 1974, pp. 144-151.

8. P. HENRICI, Discrete Variable Methods in Ordinary Differential Equations, Wiley, New York, 1962.

9. W. H. Hock, "Asymptotic expansions for multistep methods applied to nonlinear Volterra integral equations of the second kind," Numer. Math., v. 33, 1979, pp. 77-100.

10. P. A. W. Holyhead, S. McKee \& P. J. TAYlor, "Multistep methods for solving linear Volterra integral equations of the first kind," SIAM J. Numer. Anal., v. 12, 1975, pp. 698-711.

11. P. J. VAN DER Houwen, On the Numerical Solution of Volterra Integral Equations of the Second Kind, I. Stability, Report NW 42/77, Mathematisch Centrum, Amsterdam, 1977.

12. P. J. VAN DER Houwen, "Convergence and stability results in Runge-Kutta type methods for Volterra integral equations of the second kind," BIT, v. 20, 1980, pp. 375-377.

13. P. J. van Der Houwen, P. H. M. Wolkenfelt \& C. T. H. BakeR, "Convergence and stability analysis for modified Runge-Kutta methods in the numerical treatment of second kind Volterra integral equations," IMA J. Numer. Anal., v. 1, 1981, pp. 303-328.

14. J. D. LAmbert, Computational Methods in Ordinary Differential Equations, Wiley, London, 1973.

15. P. LiNZ, "Linear multistep methods for Volterra integro-differential equations," J. Assoc. Comput. Mach., v. 16, 1969, pp. 295-301.

16. J. Matтhys, “ $A$-stable linear multistep methods for Volterra integro-differential equations,” Numer. Math., v. 27, 1976, pp. 85-94. 
17. P. J. TAYLOR, "The solution of Volterra integral equations of the first kind using inverted differentiation formulae," BIT, v. 16, 1977, pp. 312-320.

18. P. H. M. Wolkenfelt, P. J. van Der Houwen \& C. T. H. BAKER, “Analysis of numerical methods for second kind Volterra equations by imbedding techniques," J. Integral Equations, v. 3, 1981, pp. 61-82.

19. P. H. M. Wolkenfelt, "Reducible quadrature methods for Volterra integral equations of the first kind," BIT, v. 21, 1981, pp. 232-241.

20. P. H. M. WolkENFELT, "The construction of reducible quadrature rules for Volterra integral and integro-differential equations.", IMA J. Numer. Anal., v. 2, 1982, pp. 131-152.

21. P. H. M. Wolkenfelt, Modified multilag methods for Volterra functional equations, Report NW 108/81, Mathematisch Centrum, Amsterdam, 1981. (Preprint.) 